\title{
El papel del bibliotecólogo en el desarrollo de Colecciones en la Biblioteca Universitaria
}

Ma. del Carmen Negrete.*

\begin{abstract}
RESUMEN
$E_{1}$ artículo presenta algunas consideraciones sobre los factores o elementos que determi nan la pla nea ción del desarrollo decolec ciones en labiblio te ca universi taria. Asimismo se hace énfasis en el papel que debe desempeñar el bibliotecólogo en el pro ce so de pla nea ción y co or di na ción del de sa rrollo de colec cio nes en bi bliotecas universitarias.
\end{abstract}

\section{ABSTRACT}

$T_{\text {his }}$ article presents some considerations regarding the factors or elements that determine the planning of the development of collections withing the university library. It also emphasizes the role pla yed by the li bra rian in the pro cess of planning and the collectiones in university libraries

Eldes ldesarrollodecoleccioneseselproceso que permite a la biblioteca construir una colección de materialesdocumentales que responde a las necesidades de información y requerimientos de servicios por los usuarios.

El término desarrollo de colecciones comenzó a manejarse y difundirse hace más de 20 años, principalmente en las bibliotecas universitarias de países anglosajones, debido a la crisis económica que se da en esa época, hecho que trajo como consecuencia el encarecimiento del material documental que frente a la baja de presupuestos forzó a los bibliotecarios a racionalizar el uso del di ne ro en la for mación de sus colecciones.

Los existen tes departamen tos deselección $y$ adquisición se vieron en la necesidad de justificar cada vez más la adquisición de materiales en función del servicio potencial que tendrían. Así, el conocimientoreal de la comunidad y la capacidad de predecir sus necesidades se convirtieron en el problema cen tral del na cien te con cep to de desarrollo de colecciones.

Por otro lado, el crecimiento desmesurado de las colecciones en este tipo de bibliotecas an tes de la cri sis eco nó mi ca y la ca rencia de espacio físico, obligó a los bibliotecarios a implementar políticas más precisas de descarte, o almacenamientos re mo tos en el me jor de los ca sos. "Am bos conceptos, otra vez en íntima relación con el uso real de los materiales y rebasando ya el concepto tradicional de selección".
Por lo anterior, los bibliotecólogos asumieron su papel en los procesos de cambio, to mandounaactitud másresponsable y comprometida en la planeación racional para el desarrollo y mantenimiento de las colecciones en sus bibliotecas. ${ }^{1}$

Autores reconocidos en el tema, como EdwardEvans, conside ranal de sa rrollo de colecciones como el proceso que permite identificar las fortalezas y debilidades de la colección de materiales de una biblioteca, en términos de las necesidades de los usuarios y recursos de la comunidad. ${ }^{2}$ Charles Osburn, a su vez, define al desarrollo de colecciones como un sistema de servicios al público, el cual se caracteriza por el proceso de toma de decisiones que determinan la conveniencia de adquirir y conservar materiales en la biblioteca. ${ }^{3}$ 
En lo mencionado por los autores, se percibe la idea de que el desarrollo de colecciones debe ser un proceso planeado, en función de los usuarios y servicios, en donde se relacione el conocimiento de la colección actual, las necesidades de información de la comunidad y los diferentes servicios que se ofrecen.

Cons truir una co lec ción en la bi blio te ca no se basa solamente en un conocimiento real de la comunidad y sus necesidades, sino también en estar al tanto de información actual y relevante de todas aquellas actividades que se realizan para satisfacer las necesidades y demandas de información, como lo es: la búsqueda bibliográfica, lase lección, la ad qui si ción, la ca talo gación, etc; así como también de las funciones y servicios que se ofrecen para llevar a cabo la accesibilidad a la información, tales como circulación, consulta, etc. Por tanto, es indudable el papel que debe jugar el bibliotecólogo en la planeación y ejecución de un programa de desarrollo de colecciones.

En toda biblioteca, independientemente de su estructura y naturaleza, la planeación del de sa rro llo de colec cio nes debe llevarse a cabo dentro del marco de la política institucional en que se inscribe. Y to mandoencuen ta aquellosfactores in ternos y externos que pudieran determinar en un momento dado la efectividad de la planeación, al comprometer con esto los servicios bibliotecarios que deben ofrecerse a una comunidad dada.

Los factores internos más comunes que orientan e influyen el desarrollo de colecciones pueden agruparse en siete grandes rubros:

Objetivo de la biblioteca

Estudiodelacomunidadespecífica

Colección actual

Recursos disponibles

Servicios que se ofrecen

Selección de materiales

Adquisiciones

Dentro de los factores externos a considerar, se pueden señalar:

Dinámica de la información

Formas de distribución

Cambios en el mercado editorial e informativo

Desarrollos tecnológicos que permiten el acceso a la información

Cooperación con otras bibliotecas.

Rose Mary Nar guile con si de ra que el de sarrollo de colecciones sólo puede ser visto como la planeación del proceso en donde se considera toda información relevante que permita tomar decisiones para una construcción racional. ${ }^{4}$ Sin embargo, reducir el concepto de desarrollo de colec- ción sólo al momento de la planeación es ignorar la riqueza del concepto y sus relaciones con miras a los servicios.

El desarrollo de colecciones implica un momento de planeación al cual se debe por principio allegar la información de todos y cada uno de los aspectos o factores apuntados para hacer operativo el objetivo del desarrollode colecciones. Peroesos factores deben someterse a un análisis y sus resultados deben constituir la base en el di se ño de es tra te gias para el lo gro de los objetivos y para la formulación de políticas de desarrollo.

Como se puede observar en la literatura bibliotecológica, el concepto desarrollo de colecciones en nuestro país se ha venido manejando hace más de una década. ${ }^{5}$ Sin embargo, su aplicación no se ha llevado a cabo en forma integral en la mayoría de las bibliotecas universitarias, debido, probablemente, a la complejidad de su planeación, a la falta de personal suficiente y a las implicaciones que se generan en la estructura organizativa de cada biblioteca.

Actualmente nuestra comunidad universitaria está más familiarizada con el poder de las nuevas tecnologías, con las diversas formas en que se presenta la información y con los diferentes tipos de servicios que permiten la accesibilidad a la información. Como consecuencia de estas visiones parciales, sustentadas más bien en la tecnología, la mayoría de las bibliotecas universitarias han descuidado la elaboración de un programa que permita desarrollar colecciones de forma racional y sistemática.

Algunos de los problemas que se evidenciaron en 1989, cuando se llevó a cabo la Mesa Re don da so bre "La se lec ción de materiales", ${ }^{6}$ fueron aquellos que manifestaron los participantes cuando señalaron que en algunas bibliotecas universitarias ciertas funciones no están consideradas al nivel de la importancia que ameritan, comolo es el man te ner un balan ce cuali tativo y cuantitativo entre las diferentes áreas de interés para la institución; así como la inexistencia de una sistematización en la formación de colecciones. Uno de los puntos de las conclusiones en dicha mesa, fue la necesidad de gestionar y dar seguimiento a la puesta en marcha de mecanismos formales para que la participación del bibliotecólogo en el desarrollo de colecciones sea un hecho.

La literatura sobre desarrollo de colecciones está enfocada básicamente en los aspectos operacionales para construir colecciones, tales como: descarte, almacenamiento, automatización, compartir recursos, etc.; por otra parte, los materiales acer ca de la evalua ción de colec cio- nes tiende a enfocarse como apoyo a los aspectos operacionales señalados. ${ }^{7}$

Lo mencionado parece apuntar sobre la necesidad de incluir, entre las variables implicadas en el desarrollo de colecciones, al factor humano relacionado con dicho proceso, cuya responsabilidad será coordinar, asignar, guiar y supervisar todas y cada una de las actividades y funciones necesarias para construir una colección.

Elprofesionalidóneopara participartan to en la pla nea ción y co or di na ción del pro ceso como en la toma de decisiones para incluir, preservar o descartar materiales, es el bibliotecólogo.

El bibliotecario ha recibido una formación profesional que le permite contar con bases teóricas para tomar decisiones. Posee una visión sobre los diversos servicios, y también la habilidad en el manejo de técnicas de acceso a la información, lo cual le permite transmitir el conocimiento a su comunidad. Además es capaz de detectar las necesidades y demandas de los usuarios; así como el estado actual de la colección en cuan to a te mas y ma te ria les que la conforman, lo cual a su vez, le otorgala capacidad para valorar los requerimientos reales en su biblioteca. Cuenta además, con importantes conocimientos del mercado editorial, en donde el problema de oferta, tiempos y costos, aunado a los factores de demandas, le permite tomar decisiones paraunóptimodesarrollodecolecciones.

Formar una colección en la biblioteca universitaria requiere de medidas que eviten tomar decisiones arbitrarias o subjetivas que no respondan a realidades concretas. Por tanto, el bibliotecólogo debe estar consciente de la necesidad de la representatividad del cuerpo académico en este proceso, no obstante ser él, el directamente responsable. Esto implicaunaparticipación conjunta si se considera que los integrantes de la comunidad tienen sus propias necesidades y que, además, ambaspartes(bibliotecólogo/comunidad)son diferentesensusniveles deespecialización $y$ en sus modalidades de trabajo.

La conceptualización de selección y no de lo que implica el desarrollo de colecciones es lo que ha llevado a considerar que son los profesores/investigadores especialistas en diferentes áreas, los únicos agentes competentes en dicho proceso, debido a su conocimiento de los materiales y de los temas que deben seleccionarse. No obstante, como se ha visto, el desarrollo de colecciones tiene que ver con problemas económicos, de mercado editorial, de visión de comportamiento de la colección en la biblioteca, etc.;información con la que no fácilmentecuentanlosprofesores/investigadores. 
Lo señalado anteriormente no puede considerarse como una generalización en todas las bibliotecas universitarias, sin embar go esta ob ser va ción tie ne su peso, pues de jar la prác ti ca del de sa rrollo de colecciones sólo al área de profesores/investigadores, significa ignorar, por ejemplo, los problemas de demandas detectadas por diferentes canales de la biblioteca, como pueden ser los servicios.

Eldesarrollodecoeccionesen labiblioteca universitaria mexicana es uno de los aspec tos más im por tan tes, ya que de ella depen de la pres ta ción de servi cios. Es al mismo tiempo, una actividad dinámica $y$ compleja a la que, en la ma yo ría de los casos, no se le presta la atención que se requiere. De ahí que la ausencia de criterios $y$ procedimientos planificados que contribuyan al ejercicio del desarrollo de coleccio nes sean prác ti ca co mún en este tipo de biblioteca.

Marcar criterios ge ne ralesparala construcción y de sa rrollo de colec cio nes y to mar la iniciativa para sugerir títulos específicos, no son as pectos ex cluyen tes, sino mo mentos de un procesoendon delosbibliote cólogos y la comunidad de la biblioteca están implicados, más que a nivel de prerrogativa al de una obligación. Por tanto, la respon sa bi li dad en la for mu la ción de políticas y criterios de desarrollo debe recaer en los bibliote cólogos, quie nessonen realidad los que tienen contacto directo con los usuarios y por ello conocen mejor sus necesidades y demandas de informa- ción general y específica; es conveniente, por otra parte, el apoyo de los profesores/investigadores especialistas en diferentes áreas, que saben cuáles son las prioridades en los programas académi$\cos ^{8}$

Otro grupo integrante de la comunidad, que no se puede perder de vista, debido a que tie ne una perma nen cia tem poral en la institución, es el de los estudiantes, los cuales, también, deben representar los intereses desu co mu nidad en la for mulación de políticas.

Para que el proceso de desarrollo de colecciones se encamine a su objetivo sin perder continuidad o caer en la parcialización, debe contemplar la existencia de una per so na que pla nee y co or di ne todo el trabajo del equipo implicado, ya que será quien finalmente haga operables las políticas de desarrollo de colecciones. Esta persona por las razones antes expuestas debe ser el bibliotecólogo, que en condiciones ideales coincidiría con la investidura de jefe de biblioteca.

Es indudable el papel que debe ju gar el bi bliotecólogo en el desarrollo de colecciones. De su formación, habilidad y experiencia profesional depende en gran medida que la colección de una biblioteca esté adecuadamente balanceada en todas las áreas de su especialidad y que los usuarios encuentren suficiente información para resolver sus problemas de estudio, de investigación y de docencia. Las cualidades necesarias para cubrir una área determinada, tanto en amplitud como en profundidad incluyen la erudición, pero también la técnica bibliográfica y el adecuado manejo de las obras de consulta.

\section{RECOMENDACIONES}

Es necesario profesionalizar a más bibliotecólogos en las labores asociadas con el desarrollo de colecciones en una perspectiva atenta a las necesidades informativas y en la perspectiva de la urgencia de la información y de los cambios en el mercado editorial e informativo.

Los res pon sables y direc tivos de las bi bliotecas universitarias deben ver la planeación del desarrollo de colecciones como una prioridad en sus bibliotecas, y deben asumir su responsabilidad frente a todo lo que implica llevar a cabo este proceso, como una tareapermanente que garantice serviciosbibliotecarioseficientesyadecuados a la comunidad universitaria.

Se pro po ne una es pe cial aten ción por parte de los bibliotecólogos al desarrollo de colecciones, en la cual no debe perderse de vista la integración de instancias que reúnan a todas las partes que tienen que ver y que se benefician con el desarrollo de colecciones, tal es la integración de los bibliotecólogos de la biblioteca universitaria con los profesores de áreas, el alum nado y los directivos.

\section{NOTAS}

1.MAGRILL, Rose Mary and John Corbin. Adquisitions management and collection development in libraries. Chicago : ALA ; 1989. p. 2

2. EVANS, Ed ward. Developinlibraryandinformationcenter. 2a. ed. Littleton, Col.: Libraries Unlimited, 1987. p. 13 (Library Sciece Text Series)

3. OSBURN, Charles B. "Toward a reconceptualization of collection development". En :Advancesinlibraryadministrationandorganization. Vol. 2 (1979) p. 177

4. MAGRILL... Op. cit. p. 16

5. OROZCOTENORIO,José. "Desarrollodecoleccionesdocumentales". En:Ciencia bibliotecaria. Vol. 3 no. 2 (1979) p. 79-81.

6. Mesa Redonda sobre "La selección de materiales documentales en bibliotecas universitariasmexicanas". Cele bradael21 dejuliode 1989 en el marcodel7o. Coloquio de Investigación bibliotecológica. Organizadopor el Cen troUniversitario de Investigaciones Bibliotecológicas de la UNAM.

7. GONZALEZ-KIRBY, Diana. "Case studies in collection development: setting an agenda for future research". En : Collection Building. vol. 11, no. 2 (1991)

8. BUIZ, Ed. "Collection developmentpolicies: coordinating tea ching fa cul ty and li brary staff in te rests at Southeast Missouri Sta te Univer sity" En:CollectionManagement vol. 13, no. 3 (1990) p. 11-17. 